\title{
Emerging Molecular Sensing Materials for Air Quality Monitoring
}

\author{
M. Bouvet ${ }^{1}$ \\ ${ }^{1}$ Institut de Chimie Moléculaire de I'Université de Bourgogne, ICMUB, UMR CNRS 6302, \\ Universitè de Bourgogne Franche-Comté, Dijon, France \\ marcel.bouvet@u-bourgogne.fr
}

\begin{abstract}
To carefully design sensing materials and to master their processing is of the utmost importance to get efficient sensors and optimize their performances for air quality monitoring (AQM). Beside inorganic materials and carbon nanomaterials, molecular materials, including macrocyclic molecules and conjugated polymers play an increasing role in the field of chemical sensors. In this mini-review devoted to molecular materials, we chose to separate sensing materials in three main categories, namely macrocyclic molecules, polymers and hybrid materials involving compounds from the two previous categories. Among macrocyclic molecules, the most important families used in chemosensing are phthalocyanines and porphyrins. Among other molecules used as gas sensing materials, cavitand compounds, e.g. resorcinarenes, offer the possibility to form inclusion complexes with volatile organic guests, which involve host-guest intermolecular interactions. Polymers used for AQM can be classified in two main categories, namely conjugated polymers, often associated with conductometric transducers and dielectric polymers in capacitive sensors. Generally speaking, the choice of the sensing materials depends not only on the target species, but also on the transduction mode, and must be envisaged according to particular applications.
\end{abstract}

Key words: Molecular materials, polymers, molecular engineering, solution processing, conductometric transducers

\section{Introduction}

A strong demand exists for gas sensors operating at low temperature and prepared with low energy processes. Thus, molecular materials, including polymeric materials, that can be often deposited by solution processing, are good candidates. Another interest for molecular materials results from the variability of the accessible materials, including hybrid organic-inorganic materials, and materials combining small molecules with carbonaceous compounds or polymers. Both the composition of the hybrid material and the parameters of the solution processing step can be used to tune the characteristics of materials, e.g. morphology, roughness and specific surface, hydrophilicity and eventually their optical and electronic properties [1]. Thus, emerging sensor technologies become available, such as ink-jet printing, which is particularly suitable for deposition on large areas and at low-cost. Besides, electrodeposition presents clear advantages, for example when different materials need to be deposited on different electrodes located on the same substrate.

\section{Molecular materials}

Among molecular materials, macrocyclic molecules like porphyrins and phthalocyanines have been highly studied as sensing materials. Due to molecular engineering, their physical properties can be tuned [2]. Thus fluorine substitution of phthalocyanines lead to n-type semiconductors whereas unsubstituted phthalocyanines exhibit a $p$-type behavior. The incorporation of ionic moieties makes them soluble in water, and the introduction of long alkyl chain allows for their solubilization in organic solvent. Among tetrapyrrole derivatives, due to their radical nature, lanthanide(III) double-decker complexes are easily oxidized and reduced, which make them good candidates to operate as sensing materials for the detection of redox active species [3]. Their particularly high conductivity allows for building various conductometric transducers, among them resistors, field-effect transistors and Molecular Semiconductors - Doped Insulators (MSDI) Heterojunctions [4]. They are built around a heterojunction between a molecular semiconductor (MS) and a doped-insulator (DI) (Fig. 1). The MS must be more conductive than 
the sub-layer to take advantage of the heterojunction. In most cases, the lutetium bisphthalocyanine $\left(\mathrm{LuPC}_{2}\right)$ is used as MS. A unique behavior is observed when the sub-layer is a n-type-material, such as fluorinated Pc, e.g. $\mathrm{Cu}\left(\mathrm{F}_{16} \mathrm{Pc}\right)$, or a perylene derivative. The energy barrier at the interface depends on the difference in energy levels of the two materials. In contrast to resistors, the MSDI exhibit nonlinear but symmetrical I(V) characteristics as a result of the energy barrier at the interface between the two layers. Even though the only material in contact with the atmosphere is a ptype material $\left(\mathrm{LuPc}_{2}\right)$, the current across MSDI increases when exposed to a donating species like $\mathrm{NH}_{3}$. Due to the particular redox properties of $\mathrm{LuPC}_{2}$, the MSDls are very sensitive. Donating species induces a decrease of majority charge carriers, leading to a current decrease in p-type resistors, whereas their effect is inverted in n-MSDI where the electronic transport is governed by minority charge carriers [5]. The better stability towards humidity was obtained with $\mathrm{Cu}\left(\mathrm{F}_{16} \mathrm{Pc}\right)$ as a n-type sublayer. Thus, for $\mathrm{Cu}\left(\mathrm{F}_{16} \mathrm{PC}\right) / \mathrm{LuPC}_{2}$ MSDI (50 $\mathrm{nm} / 50 \mathrm{~nm})$, the relative response to $\mathrm{NH}_{3}$ remains unaffected by the variation of relative humidity in the $20-80 \%$ range. Moreover, this response has proved to be stable over time, for several years.

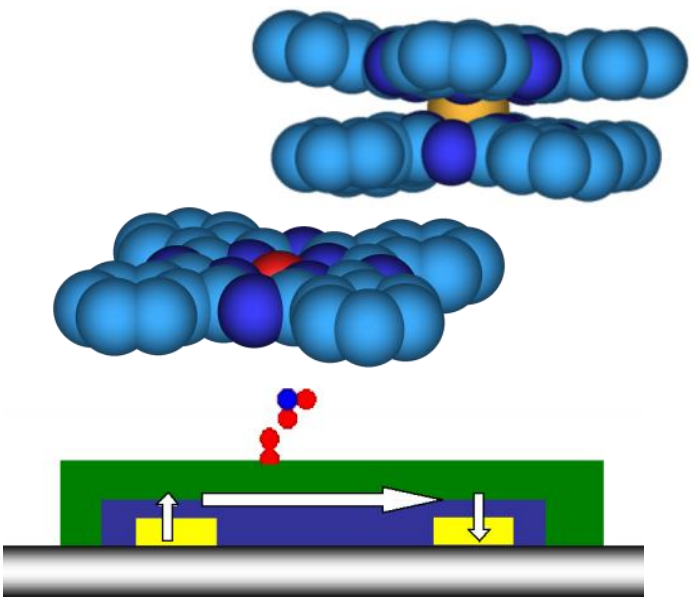

Fig. 1. From top to bottom: $\mathrm{LnPC}_{2}$ and $\mathrm{MPC}$ phthalocyanine complex and an MSDI device; the arrows indicate the charge transport pathway.

We also studied a n-MSDI made from the 2,2,3,3,4,4,4-heptafluorobutyl-perylene-bisimide ( $\mathrm{C}_{4} \mathrm{~F}_{7}$-PTCDI), n-type sub-layer [6]. We exposed the MSDI to ammonia in the 10-90 ppm range. This MSDI exhibits an increase of $\mathrm{I}$, with a relative response $\mathrm{RR}=\left(\mathrm{I}_{\mathrm{on}}-\mathrm{I}_{\text {off }}\right) / \mathrm{I}_{\text {off }}$ of 33 $\%$ at $90 \mathrm{ppm}$. At $10 \mathrm{ppm}$, the RR is still of $6 \%$. The RR variation as a function of the $\mathrm{NH}_{3}$ concentration is not linear but may be fitted by a polynomial (Fig. 2).

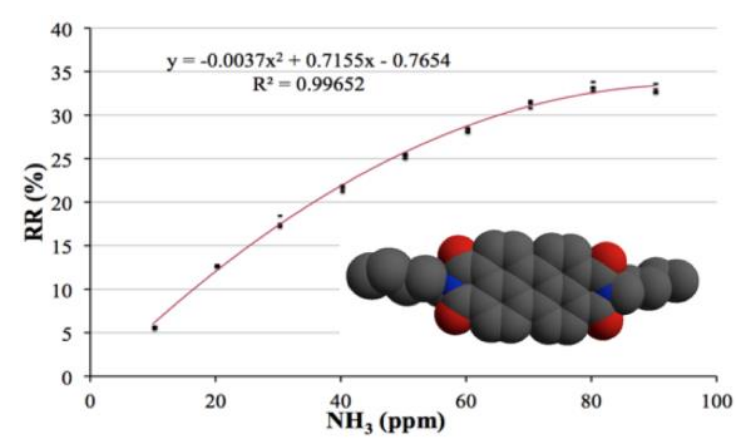

Fig. 2. Relative response of a $C_{4} F_{7}-P T C D I / L U P C_{2}$ MSDI exposed to $10-90$ ppm of ammonia under 50 $\% \mathrm{rh}$, and a view of the perylene derivative ( $F$ and $H$ atoms have been omitted).

Different solution processing techniques can be used to prepare thin films of molecular materials, from the very simple solvent cast to the quasi Langmuir-Shäfer technique developed for the deposition of an amphiphilic tris(phthalocyaninato) europium triple decker complex [7]. Spin-coating was also used to prepare hybrid films containing hydroxygallium phthalocyanine and cellulose, starting from a trimethylsilyl derivative [8]. The kinetics of the chemosensing process was highly related to the film morphology, as shown by the change in the surface potential under cyclic exposure to ozone and nitrogen.

\section{Cavitands}

Among other molecules used as gas sensing materials, cavitand compounds offer the possibility to form inclusion complexes with volatile organic guests. At a first glance, the size of the cavity and its hydrophobic/hydrophilic character should be the two key parameters responsible for the selectivity of gas sensors (Fig. 3). However, studies achieved with amphiphilic calix[4]resorcinarene derivatives deposited by the LB technique showed that the adsorption ability depends on the condensed vapor pressures of the adsorbates, rather than on a structural coincidence between host cavities and guest molecules [9]. The results obtained from the isotherms of adsorption determined by both QCM and Surface Plasmon Resonance (SPR) were interpreted in terms of capillary condensation of organic vapors in the nanoporous matrix of cavitand-containing films. Ellipsometric measurements showed changes of both the thickness and refractive index of the films caused by adsorption. Films exposed to a range of concentrations of the vapors of benzene, toluene, n-hexane, m-xylene, and 1butanol showed that their responsivity was 
correlated with the solubility of the sensing material in these solvents [9].
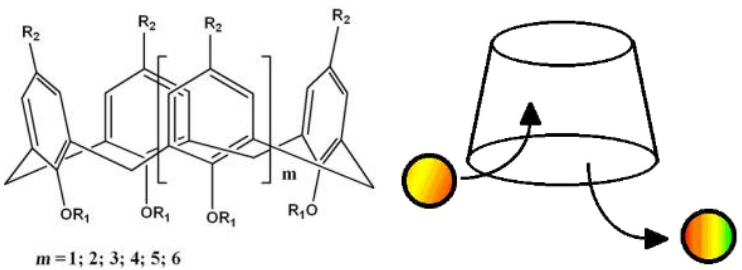

Fig. 3. Schematic view of calixarenes as examples of cavitand molecules used for the detection of VOCs, and the principle of host-guest recognition.

This apparent selectivity was directly related to the vapor pressure of the VOCs. Thus, in the case of BTX, the response was higher for xylene than for toluene and benzene, associated to a higher vapor pressure for xylene. However, recently, a nice example of selectivity towards benzene was obtained with covalently attached quinoxaline-bridged resorcin[4]-arene cavitands to gold nanoparticles anchored on oxygen plasma treated carbon nanotubes [10]. In this case, the response to benzene was very high compared to other studied VOCs, including toluene and xylene.

\section{Polymers}

Two effects peculiar to polymers can change their physical properties. At first, the swelling that generally induces a decrease of their conductivity value [11, 12], and also the effect of temperature that can highly change the motion of polymer chains segments. Thus, gas diffusion is faster in low glass transition $\left(T_{g}\right)$ polymers, or when the operating temperature lies above the $T_{g}$ of the sensing polymer [13]. The addition of plasticizers, e. g. phthalate derivatives, can be used to introduce a decrease of $\mathrm{Tg}$, then a higher sensitivity to gases. The interaction of polymers with VOCs can be described using linear solvation energy relationships (LSERs) that take into account dispersion, polarizability, dipolarity, basicity, acidity and hydrogen bonding interactions [14]. Polyimide, cellulose acetate, polycellulose acetate butyrate, polymethylmethacrylate and polyvinylpyrrolidone, polyethylene-naphthalate are known as dielectric polymers, and used as sensing materials in capacimetric humidity sensors (Fig. 4).

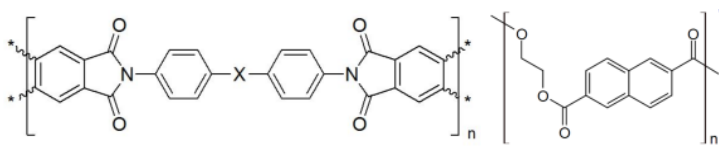

Fig. 4. Polyimide and polyethylene naphthalate as examples of dielectric polymers used in relative humidity sensors.
Conjugated polymers, as polypyrrole, polyaniline, polythiophene, but also other conducting polymers generally used in organic electronics, have been highly used in conductometric transducers. As other conducting materials, they are first sensitive to redox active species like nitrogen dioxide and ozone, well-known oxidizing pollutants (Fig. 5). They can generally be synthesized by chemical or electrochemical oxidation of the corresponding monomers. As soon as they are soluble, they can be deposited by some solution processing, namely spin-coating, dipcoating, drop-coating, Langmuir-Blodgett technique or layer-by-layer technique [15]. However, the advantage of the electrochemical method is to ensure the synthesis and the deposition process in a unique step.<smiles></smiles>

Fig. 5. Polypyrrole and one of its partially oxidized forms as an example of conducting polymer used in conductometric transducers.

\section{Polymer-macrocycle hybrid materials}

In the case of conducting polymer/ phthalocyanine hybrid materials, again different solution processing techniques can be used, as the layer-by-layer and electrochemical techniques, both operated at room temperature. They can lead to highly performant gas sensors, as illustrated by their response to ammonia in a broad range of relative humidity.

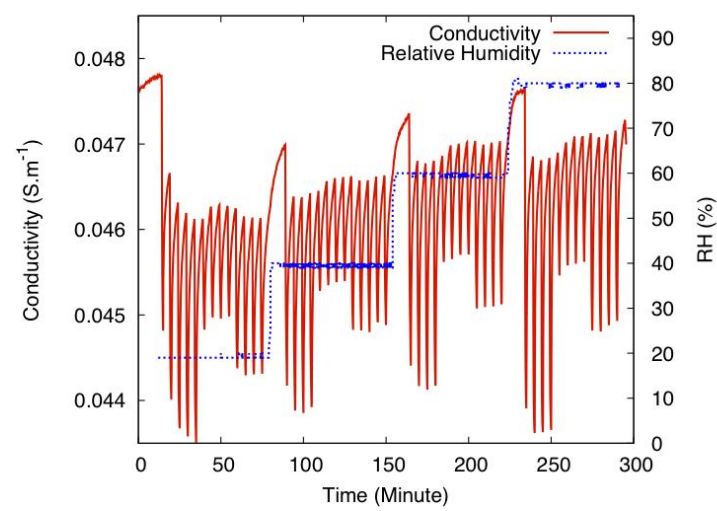

Fig. 6. Conductivity variation of a resistor made from polypyrrole and a sulfonated cobalt phthalocyanine exposed to 25, 45, and $90 \mathrm{ppm}$ of ammonia under relative humidity in range $20-80 \%$. 
Thus, a polypyrrole film electrodeposited in the presence of an anionic metallophthalocyanine exhibits a very different morphology than those deposited with small counter anions, leading to a higher sensitivity (Fig. 6) [16, 17]. Solution processing can be performed even in water, as exemplified by the layer-by-layer deposition of polyaniline - tetrasulfonated phthalocyanine hybrid films [18].

\section{Acknowledgements}

The author acknowledges the Agence Nationale de la Recherche (ANR, France) for funding through the ANR projects CAP-BTX 2010 and OUTSMART 2015. Financial support from the European Union and the Conseil Régional de Bourgogne through the FABER and the PARI SMT 08 and CDEA programs is gratefully acknowledged. We would like to thank the European Union for fundings (FEDER and short term scientific missions through the COST Action TD1105 EuNetAir).

\section{References}

[1] M. Bouvet, A. Pauly, Molecular Semiconductor Based Gas Sensors, in The Encyclopedia of Sensor, Eds. C. A. Crimes, E. C. Dickey and M. Pishko, American Scientific Publishers, Stevenson Ranch, vol. 6, 227-270, 2006.

[2] Y. Chen, D. Li, N. Yuan, J. Gao, R. Gu, G. Lu, M. Bouvet, Tuning the Semiconducting Nature of Bis(phthalocyaninato) Holmium Complexes via Peripheral Substituents, J. Mater. Chem. 22 22142-22149 (2012); doi: 10.1039/C2JM35219B

[3] M. Bouvet, Radical Phthalocyanines and Intrinsic Semiconduction, in The Porphyrin Handbook, Eds. K. M. Kadish, K. M. Smith, R. Guilard, Academic Press, vol 19, 37-103, 2003.

[4] V. Parra, J. Brunet, A. Pauly, M. Bouvet, Molecular semiconductor - doped insulator (MSDI) heterojunctions, an alternative transducer for gas chemosensing, Analyst, 1776-1778 (2009); doi: 10.1039/B906786H

[5] M. Bouvet, P. Gaudillat, A. Kumar, T. Sauerwald, M. Schüler, A. Schütze, J.-M. Suisse, Revisiting the electronic properties of Molecular Semiconductor - Doped Insulator (MSDI) heterojunctions through impedance and chemosensing studies, Org. Electron. 26, 345354 (2015); doi: 10.1016/j.orgel.2015.07.052

[6] P. Gaudillat, A. Wannebroucq, J.-M. Suisse, M. Bouvet, Bias and humidity effects on the ammonia sensing of perylene derivative/lutetium bisphthalocyanine MSDI heterojunctions, Sens. Actuators: B. Chemical 222, 910-917 (2016); doi: 10.1016/j.snb.2015.09.015

[7] Y. Chen, M. Bouvet, T. Sizun, Y. Gao, C. Plassard, E. Lesniewska, J. Jiang, Facile Approaches to build Ordered Amphiphilic Tris(phthalocyaninato) Europium Triple-Decker
Complex Thin Films and their comparative performances in ozone sensing, Phys. Chem. Chem. Phys. 12, 12851-12861 (2010); doi: 10.1039/COCP00289E

[8] V. Parra, M. Rei Vilar, A. M. Botelho Rego, A. M. Ferraria, S. Boufi, M. L. Rodriguez-Mendez, E. Fonavs, I. Muzikante, M. Bouvet, Novel hybrid films based on cellulose/hydroxygallium phthalocyanine. Spectroscopic, surface and gas sensing studies, Langmuir 23, 3712-3722 (2007); doi: 10.1021/la063114i

[9] A. V. Nabok, A. K. Hassan, A. K. Ray, O. Omar, V. I. Kalchenko, Study of adsorption of some organic molecules in calix[4]resorcinolarene LB films by surface plasmon resonance, Sens. Actuators: B. Chemical 45, 115-121 (1997); doi: 10.1016/S0925-4005(97)00282-7

[10] P. Clément, S. Korom, C. Struzzi, E. J. Parra, C. Bittencourt, P. Ballester, E. Llobet, Benzene Detection: Deep Cavitand Self-Assembled on Au NPs-MWCNT as Highly Sensitive Benzene Sensing Interface, Adv. Funct. Mater. 25, 40114020 (2015); doi: 10.1002/adfm.201570180

[11] R. A. Potyrailo, C. Surman, N. Nagrajand, A. Burns, Materials and Transducers Toward Selective Wireless Gas Sensing, Chem. Rev. 111, 7315-7354 (2011); doi: 10.1021/cr2000477

[12] U. Altenberend, A. Oprea, N. Barsan, U. Weimar, Contribution of polymeric swelling to the overall response of capacitive gas sensors, Anal. Bioanal. Chem. 405, 6445-6452 (2013); doi: 10.1007/s00216-013-7023-x

[13] D. T. McQuade, A. E. Pullen, T. M. Swager, Conjugated Polymer-Based Chemical Sensors, Chem. Rev. 100, 2537-2574 (2000); doi: $10.1021 / \mathrm{cr} 9801014$

[14] J. W. Grate, Hydrogen-Bond Acidic Polymers for Chemical Vapor Sensing, Chem. Rev. 108, 726745 (2008); doi: 10.1021/cr068109y

[15] D. Nicolas-Debarnot, F. Poncin-Epaillard, Polyaniline as a new sensitive layer for gas sensors, Analytica Chimica Acta 475, 1-15 (2003); doi: 10.1016/S0003-2670(02)01229-1

[16] S. Carquigny, J.-B. Sanchez, F. Berger, B. Lakard, F. Lallemand, Ammonia gas sensor based on electrosynthesized polypyrrole films, Talanta 78, 199-206 (2009); doi: 10.1016/j.talanta.2008.10.056

[17] T. Sizun, T. Patois, M. Bouvet, B. Lakard, Microstructured electrodeposited polypyrrolephthalocyanine hybrid material, from morphology to ammonia sensing, J. Mater. Chem. 22, 2524625253 (2012); doi: 10.1039/C2JM35356C

[18] P. Gaudillat, F. Jurin, B. Lakard, C. Buron, J.-M. Suisse, M. Bouvet, From the Solution Processing of Hydrophilic Molecules to Polymer-Phthalocyanine Hybrid Materials for Ammonia Sensing in High Humidity Atmosphere, Sensors 14, 1347613495 (2014); doi: 10.3390/s140813476 